\title{
Strong Coupling Corrections in Quantum Thermodynamics
}

\author{
M. Perarnau-Llobet, ${ }^{1,2}$ H. Wilming, ${ }^{3}$ A. Riera, ${ }^{1,2}$ R. Gallego, ${ }^{3}$ and J. Eisert ${ }^{3}$ \\ ${ }^{1}$ Max-Planck-Institut für Quantenoptik, D-85748 Garching, Germany \\ ${ }^{2}$ ICFO-Institut de Ciencies Fotoniques, The Barcelona Institute of Science and Technology, 08860 Castelldefels, Barcelona, Spain \\ ${ }^{3}$ Dahlem Center for Complex Quantum Systems, Freie Universität Berlin, 14195 Berlin, Germany
}

(Received 21 May 2017; revised manuscript received 19 December 2017; published 22 March 2018)

\begin{abstract}
Quantum systems strongly coupled to many-body systems equilibrate to the reduced state of a global thermal state, deviating from the local thermal state of the system as it occurs in the weak-coupling limit. Taking this insight as a starting point, we study the thermodynamics of systems strongly coupled to thermal baths. First, we provide strong-coupling corrections to the second law applicable to general systems in three of its different readings: As a statement of maximal extractable work, on heat dissipation, and bound to the Carnot efficiency. These corrections become relevant for small quantum systems and vanish in first order in the interaction strength. We then move to the question of power of heat engines, obtaining a bound on the power enhancement due to strong coupling. Our results are exemplified on the paradigmatic nonMarkovian quantum Brownian motion.
\end{abstract}

DOI: 10.1103/PhysRevLett.120.120602

Thermodynamics is the fundamental theory concerned with heat and temperature and their relation to energy and work. In phenomenological thermodynamics, an implicit assumption is that couplings between the working systems and their heat baths are so weak so that effects of the interaction can be neglected. As a consequence, the equilibrium states of the working systems are thermal states, in fact thermal states of local Hamiltonians. For small-scale systems governed by quantum mechanical laws, however, such a weak-coupling limit can be far from being reasonable, as the surface area of such systems is often not much smaller than their volume. An impressive body of literature in a related field, namely, equilibration and thermalization of closed quantum many-body systems [1], strongly suggests that a system coupled strongly to a thermal bath should be described by the local reduced state of the global Gibbs state $\rho_{S}=\operatorname{Tr}_{B}\left[e^{-\beta H} / \operatorname{tr}\left(e^{-\beta H}\right)\right]$ - and not by a Gibbs state of the local Hamiltonian itself [2-5].

In this work we take this basic but profound insight seriously when studying in detail quantum thermal machines strongly coupled to heat baths. First, we prove exact and general bounds on work extraction from a nonequilibrium system that can be brought in contact with a single heat bath. These results can be captured as universal corrections to the weak coupling limit-showing that strong coupling unavoidably leads to irreversibility and is hence detrimental for work extraction. Similar corrections are obtained for heat dissipation and the Carnot efficiency, hence providing strong-coupling corrections to the different formulations of the second law of thermodynamics. For thermal machines, we also show that strong interactions lead to power enhancements.

There have been recent efforts to describe the thermodynamics of quantum systems with strong interactions between system and bath [6-37]. These include considerations on heat engines [9-14], equilibrium and nonequilibrium thermodynamics [15-30,33-37]. The key contribution of the present work, compared with earlier strong-coupling analyses [9-14,21], is to provide bounds on work and efficiency, without having to restrict to any particular model for the systems involved. Our bounds apply to thermodynamic scenarios in which the system equilibrates to the reduced state of a global Gibbs state, and for which the coupling can be switched on and off. More precisely, our results are derived within a framework applicable to general situations; after all, also phenomenological thermodynamics is widely applicable by largely abstracting from the specifics of a given setting.

Framework.-We consider a system $S$, a heat bath $B$, with internal Hamiltonians $H_{S}$ and $H_{B}$, respectively. They can interact via a possibly strong interaction $V$. Thermodynamic protocols then consist on transformations over $H_{S}$, and equilibration processes induced by $V$. Specifically, we consider protocols of $N$ steps, and denote by $\rho^{(i)}$ and $H^{(i)}$ the state and Hamiltonian of $S B$ in the $i$ th step, consisting of three elementary operations:

(A) Turning on and off interaction.-With this, we model the process of bringing $S$ and $B$ into contact, so that the Hamiltonian takes the form $H^{(i)}=H_{S}^{(i)}+H_{B}+V$. Similarly, $V$ can be turned off at any step of the process. Treating such processes as quenches, the average work gain when placing or removing $V$ is

$$
W_{\mathrm{on}}^{(i)}=\operatorname{Tr}\left(\rho^{(i)} V\right)=-W_{\mathrm{off}}^{(i)} .
$$

(B) A quench on S.-A fast transformation of $H_{S}$ is implemented, so that $H^{(i)}=H_{S}^{(i)}+H_{B}+V$ is changed to 
$H^{(i+1)}=H_{S}^{(i+1)}+H_{B}+V$, whereas the state $\rho^{(i)}$ remains unchanged. The corresponding work gain reads

$$
W^{(i)}=\operatorname{Tr}\left[\rho_{S}^{(i)}\left(H_{S}^{(i)}-H_{S}^{(i+1)}\right)\right],
$$

which depends only on the state of $S$ [38].

(C) A thermalization process.-This operation models the closed free evolution of $S B$ when $V$ is present, i.e., under $H^{(i+1)}$. This operation has no work cost, as the total energy of $S B$ is preserved. When they reach equilibrium, we assume that $S$ is well described by

$$
\rho_{S}^{(i+1)}=\operatorname{Tr}_{B}\left[\omega_{\beta}\left(H^{(i+1)}\right)\right],
$$

where $\omega_{\beta}(H)=e^{-\beta H} / \operatorname{tr}\left(e^{-\beta H}\right)$.

Similarly, we assume that the boundary between $S$ and $B$, i.e., the support of $V$, can also be described by the reduced form of $\omega_{\beta}\left(H^{(i+1)}\right)$. Both assumptions are reasonable for locally interacting systems and are backed by a body of rigorous arguments $[1,5,39]$. When it is clear from the context we will use the notation $\omega^{(i)}:=\omega_{\beta}\left(H^{(i)}\right)$ and $\omega_{S}^{(i)}:=\omega_{\beta}\left(H_{S}^{(i)}\right)$. We also use the convention $\hbar=1$, $k_{B}=1$, and that when $S B$ decrease their global energy, then work $W>0$ is extracted.

A thermodynamic protocol then consists of an arbitrary sequence of operations of the types (A)-(C). The total expected work $W$ gained in the process is the sum of all the contributions of the form (1) and (2). In this framework, the Hamiltonian terms $V$ and $H_{B}$ remain fixed throughout the protocol, reflecting the fact that an experimenter will in many realistic situations not have precise control over $B$ and the coupling between $S$ and $B$, at least not beyond the capability of turning it on and off. During transformations of the form (C), $S$ is assumed to be brought to equilibrium after sufficiently long times. That is, possible finite-time effects are not included.

Maximal work extraction for arbitrary coupling strengths. - We now study work extraction from an outof equilibrium state of $S$. In order to avoid the possibility of extracting work from the energy stored in $V$, we consider that $S$ is initially isolated from $B$. The initial Hamiltonian is hence noninteracting, $H^{(0)}=H_{S}+H_{B}$, and the initial state is uncorrelated, $\rho^{(0)}=\rho_{S} \otimes \omega_{\beta}\left(H_{B}\right)$. Given these initial conditions, the task is to optimize the extracted work over all cyclic Hamiltonian processes under the operations (A)-(C) [42].

It is instructive to first recall the optimal protocol in the weak-coupling regime [22,43-45]. It consists of four steps: (i) a quench from $H_{S}^{(0)}$ to $\tilde{H}_{S}$, where $\omega_{\beta}\left(\tilde{H}_{S}\right)=\rho_{S}$, (ii) turning on $V$, (iii) an isothermal process from $\tilde{H}_{S}$ back to $H_{S}$, and (iv) turning off $V$. In our framework, isothermal processes correspond to a concatenation of infinitesimally small quenches followed by equilibration steps-see
Refs. [22,45,46] for more details. The protocol (i)-(iv) has no dissipation, and is hence reversible in the limit of an arbitrarily weak $V$. In the strong-coupling regime, we show in Sec. III of the Supplemental Material (SM) [46] that the optimal protocol also has the form (i)-(iv), but the initial and final Hamiltonians of the isothermal process need to be modified. Let $H_{S}^{(1)}$ and $H_{S}^{(N)}$ be the Hamiltonians of $S$ when $V$ is turned on and off, respectively. Then, the protocol's total work $W$ reads

$$
W=W^{(\text {weak })}-\Delta F^{(\text {res })}-\Delta F^{(\text {irr })},
$$

where $W^{(\text {weak })}=F\left(\rho_{S}, H_{S}\right)-F\left(\omega_{\beta}\left(H_{S}\right), H_{S}\right)$ is the maximal extractable work in the weak coupling regime, $F(\rho, H):=\operatorname{Tr}(\rho H)+T \operatorname{Tr}(\rho \ln \rho)$ is the (nonequilibrium) free energy, and we have defined

$$
\begin{aligned}
\Delta F^{(\mathrm{irr})} & :=F\left(\rho^{(0)}, H^{(1)}\right)-F\left(\omega^{(1)}, H^{(1)}\right), \\
\Delta F^{(\mathrm{res})} & :=F\left(\omega^{(N)}, H^{(0)}\right)-F\left(\omega^{(0)}, H^{(0)}\right),
\end{aligned}
$$

with $H^{(1) /(N)}=H_{S}^{(1) /(N)}+H_{B}+V$. Note that $F(\rho, H)-$ $F\left[\omega_{\beta}(H), H\right]=T S\left[\rho \| \omega_{\beta}(H)\right] \geq 0 \quad$ with $\quad \mathrm{S}(\rho \| \sigma):=$ $\operatorname{Tr}[\rho(\log \rho-\log \sigma)]$ the quantum relative entropy. It follows that always $\Delta F^{\text {(irr)/(res) }} \geq 0$, and we can already conclude that strong coupling cannot be beneficial for work extraction as $W \leq W^{\text {(weak) }}$. The correcting term $\Delta F^{(\text {irr })}$ can be interpreted as the energy dissipated when $S$ is put in contact with $B$, whereas $\Delta F^{(\text {res })}$ is the extractable work left on the final state. The extracted work $W$ in Eq. (4) is maximized when $H_{S}^{(1)}$ and $H_{S}^{(N)}$ minimize the correcting terms $\Delta F^{(\text {irr })}$ and $\Delta F^{\text {(res) }}$, respectively. Assuming that $\rho_{S}$ has full rank, we show in Sec. II of the Supplemental Material [46] that this happens for

$$
\begin{gathered}
\operatorname{Tr}_{B}\left[\omega_{\beta}\left(H^{(1)}\right)\right]=\rho_{S}, \\
\operatorname{Tr}_{B}\left[\omega_{\beta}\left(H^{(N)}\right)\right]=\frac{\operatorname{Tr}_{B}\left(\omega_{\beta}\left(H^{(N)}\right)\left(H_{H^{(N)}, \beta}^{(0)}-H^{(N)}\right)\right)}{\operatorname{Tr}\left(\omega_{\beta}\left(H^{(N)}\right)\left(H_{H^{(N)}, \beta}^{(0)}-H^{(N)}\right)\right)},
\end{gathered}
$$

where $Y_{H, \beta}:=\int_{0}^{1} d \tau e^{\beta \tau H} Y e^{-\tau \beta H}$ for Hermitian operators $Y$ - an integral that can be solved analytically; see Sec. II B of the Supplemental Material [46]. Furthermore, $\Delta F^{\text {(irr)/(res) }}$ have at least one minimum, so that Eqs. (7), (8) always provide the desired solution; see Sec. III of the Supplemental Material [46]. Altogether, our techniques provide a procedure to determine for any model the optimal protocol for work extraction in the strong coupling regime. Essentially, it consists of an isothermal process, where $S$ is put in contact with $B$ according to Eqs. (7) and (8).

It is important to stress that although a priori Eqs. (7), (8), and in general $W$, depend on the entire bath $B$, 
commonly its Hamiltonian is local and the correlations between its degrees of freedom decay rapidly with the distance. Therefore, only the degrees of freedom that are geometrically close to the boundary between $S$ and $B$ will contribute. This has the important consequence that we can solve Eqs. (7), (8) by considering a small buffer region in $B$, while maintaining tight bounds for the error made in such a prescription $[40,59,60]$. This renders the solution practically and efficiently computable.

Corrections at lowest order of work.-Interestingly, in a perturbative treatment, the problem at hand can be essentially solved by computing covariances. Let us replace $V$ by $g V$, where the dimensionless $g>0$ quantifies the interaction strength. In Sec. III C of the Supplemental Material [46], Eqs. (7) and (8) are expanded in $g$, obtaining

$$
\begin{aligned}
& H_{S}^{(1)}=\tilde{H}_{S}-g \operatorname{Tr}_{B}\left[\rrbracket_{S} \otimes \omega\left(H_{B}\right) V\right]+O\left(g^{2}\right), \\
& H_{S}^{(N)}=H_{S}-g \operatorname{Tr}_{B}\left[\rrbracket_{S} \otimes \omega\left(H_{B}\right) V\right]+O\left(g^{2}\right),
\end{aligned}
$$

where we recall that $\tilde{H}_{S}$ satisfies $\rho_{S}=\omega_{\beta}\left(\tilde{H}_{S}\right)$. Inserting Eqs. (9) and (10) into $\Delta F^{\text {(irr) }}$ and $\Delta F^{\text {(res) }}$ respectively, provides $\Delta F_{\min }^{(\mathrm{irr} /(\text { res })}:=\min _{H_{S}^{(1) /(N)}} \Delta F^{(\mathrm{irr}) /(\text { res })}$ at lowest nonvanishing order in $g$ (See Sec. III C of the Supplemental Material [46] for details.)

$$
\Delta F_{\min }^{(\mathrm{irr})}=\frac{\beta g^{2}}{2} \operatorname{cov}_{\omega_{\beta}\left(\tilde{H}^{(0)}\right)}(\tilde{V}, \tilde{V})+O\left(g^{3}\right),
$$

and, similarly, $\Delta F_{\min }^{(\mathrm{res})}=\beta g^{2} \operatorname{cov}_{\omega_{\beta}\left(H^{(0)}\right)}(\tilde{V}, \tilde{V}) / 2+O\left(g^{3}\right)$. Here, we have defined $\tilde{V}:=V-\operatorname{Tr}_{B}\left[V \omega_{\beta}\left(H_{B}\right)\right]$, $\tilde{H}^{(0)}:=\tilde{H}_{S}+H_{B}$, and $\operatorname{cov}_{\omega_{\beta}(H)}(A, B)=\operatorname{Tr}\left[A_{H, \beta} B \omega_{\beta}(H)\right]-$ $\operatorname{Tr}\left[A \omega_{\beta}(H)\right] \operatorname{Tr}\left[B \omega_{\beta}(H)\right]$ is the generalized covariance $[40,46]$, also known as the Kubo-Mori inner product [47-49].

Some important remarks are now in order. (i) The expansion of $\Delta F_{\min }^{(\mathrm{irr}) /(\mathrm{res})}$ at order $\mathcal{O}\left(g^{2}\right)$ depends only on $H^{(1) /(N)}$ at order $\mathcal{O}(g)$, as shown in Sec. IIIC of the Supplemental Material [46]. (ii) The first order correction to $W$ vanishes for any $H_{S}^{(1)}=\tilde{H}_{S}+O(g), H_{S}^{(N)}=H_{S}+O(g)$. This follows from the penalty terms $\Delta F^{(\text {irr }) /(\text { res })}$ being differentiable functions of $g$ and having a minimum at $g=0$. The choice (9), (10) provides the minimum coefficient of $O\left(g^{2}\right)$. (iii) The first order correction in Eqs. (9), (10) exactly compensates for the term $g \operatorname{Tr}_{B}\left[\omega\left(H_{B}\right) V\right]$, which often appears in open quantum systems as an effective action of $B$ on $S$ [61,62]. (iv) The generalized covariance $\operatorname{cov}_{\omega_{\beta}(H)}(A, B)$ captures the linear response of the thermal state under perturbations [47-49].

Heat and dissipation.-Let us now turn to heat dissipation in an isothermal process in the strong coupling regime. For that, we do not consider a cyclic process, and we instead fix the initial and final Hamiltonian to be $H_{S}$ and
$H_{S}^{(N)}$, respectively [63]. We consider the same initial state as in the work-extracting protocol, i.e., $\rho^{(0)}=$ $\rho_{S} \otimes \omega_{\beta}\left(H_{B}\right)$.

From the first law of thermodynamics, the total heat reads $\quad Q=\Delta E_{S}+W$, with $\quad \Delta E_{S}=\operatorname{Tr}\left(H_{S}^{(N)} \omega^{(N)}\right)-$ $\operatorname{Tr}\left(H_{S} \rho^{(0)}\right)$. Since $\Delta E_{S}$ is fixed by $\rho_{S}$ and $H_{S}^{(N)}$, it becomes clear that the protocol for maximizing $W$ also minimizes dissipation $-Q$. Then, from Eq. (4), we obtain (see Sec. IV of the Supplemental Material [46])

$$
Q=T \Delta S-\left[\Delta F_{B}^{(\mathrm{res})}+T I\left(\omega^{(N)} ; S: B\right)+\Delta F^{(\mathrm{irr})}\right],
$$

where $\Delta S=S\left[\operatorname{Tr}_{B}\left(\omega^{(N)}\right)\right]-S\left(\rho_{S}\right)$ is the gain of entropy of $S, \Delta F_{B}^{(\mathrm{res})}=T\left[\operatorname{Tr}_{S}\left(\omega^{(N)}\right) \| \omega_{\beta}\left(H_{B}\right)\right]$ is the increase of the free energy of $B$, and $I\left(\omega^{(N)} ; S: B\right)>0$ is the mutual information between $S$ and $B$. In the strong coupling case, $Q<T \Delta S$, even when the isothermal process is accomplished reversibly. Again, this is due to the penalizing terms $\Delta F_{B}^{(\text {res })}$ and $\Delta F^{\text {(irr) }}$, in addition to the correlations captured by the mutual information. Minimizing dissipation corresponds to minimizing the negative terms in Eq. (12). However, here $\Delta F_{B}^{(\text {res })}$ and $I\left(\omega^{(N)} ; S: B\right)$ are fixed through $H^{(N)}$. Hence, we only have freedom to minimize $\Delta F^{(\text {irr })}$, a problem that has been solved in Eq. (7).

Similar to the case of work, we can expand the correcting terms over the interaction strength $g$. As before, the first order correction vanishes, so that the series expansion reads

$$
Q=T \Delta S-K_{q} g^{2}+O\left(g^{3}\right),
$$

with $K_{q}>0$, and where we note that $\Delta S$ depends on $\omega_{\beta}\left(H^{(N)}\right)$ and, hence, indirectly also on $g$ [64]. From Eq. (12), the simple and useful lower bound $K_{q} \geq$

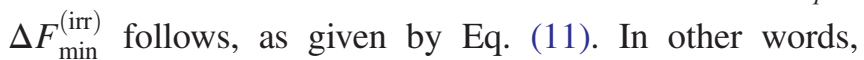
Eq. (11) also provides a strong coupling correction to dissipation, and to the Clausius formulation of the second law.

Heat engines.-Given Eqs. (4) and (12), bounds to the efficiency of a heat engine can be extended to the strong coupling regime. We consider engines made up of two baths at different temperatures which can sequentially interact strongly with $S$. We extend our formalism to account for equilibrations of the form (3) with two baths $B_{c}$ or $B_{h}$ at two different (inverse) temperatures $\beta_{c}$ and $\beta_{h}$. The task is then to maximize the efficiency of a cycle of the engine. The optimal cycle turns out to have the same form as a Carnot engine (see Sec. VA of the Supplemental Material [46]), consisting of (i) an isothermal transformation in contact with $B_{h}$ from $H_{S}^{(A)}$ to $H_{S}^{(B)}$, (ii) a quench from $H_{S}^{(B)}$ to $H_{S}^{(C)}$, (iii) an isothermal transformation with $B_{c}$ from $H_{S}^{(C)}$ to $H_{S}^{(D)}$, and (iv) a quench from $H_{S}^{(D)}$ back to $H_{S}^{(A)}$. For weak coupling, the efficiency is maximized 
through the choice of Hamiltonians $\omega_{\beta_{h}}\left(H_{S}^{(B) /(A)}\right)=$ $\omega_{\beta_{c}}\left(H_{S}^{(C) /(D)}\right)$, guaranteeing no dissipation. Given our previous results, these conditions are naturally extended for strong coupling to $\operatorname{Tr}_{B_{h}} \omega_{\beta_{h}}\left(H^{(B) /(A)}\right)=$ $\operatorname{Tr}_{B_{c}} \omega_{\beta_{c}}\left(H^{(C) /(D)}\right)$, where $H^{(X)}:=H_{S}^{(X)}+V+H_{B}$. This provides a simple recipe for constructing minimal dissipation engines in the strong coupling regime. We show in Sec. V of the Supplemental Material [46] that the corresponding maximal efficiency $\eta$, using $\eta=1-\left|Q_{c}\right| /\left|Q_{h}\right|$, (13) and expanding in $g$, reads

$$
\eta=\eta^{\mathrm{C}}-g^{2} \frac{T_{c}}{T_{h}}\left(\frac{K_{q}^{(h)}}{Q_{h}^{(\text {weak })}}+\frac{K_{q}^{(c)}}{Q_{c}^{(\text {weak })}}\right)+O\left(g^{3}\right),
$$

where $\eta^{C}$ is the Carnot efficiency, $Q_{h / c}^{(\text {weak })}=T_{h / c} \Delta S^{(\text {weak })}$ with $\Delta S^{(\text {weak })}=S\left(\omega_{\beta}\left(H_{S}^{(B)}\right)\right)-S\left(\omega_{\beta}\left(H_{S}^{(D)}\right)\right)$ and $K_{q}^{(h / c)}$ are coefficients obtained from Eq. (13) for $B_{h / c}$. By recalling the bound $K_{q} \geq \Delta F_{\min }^{(\mathrm{irr})}$ [at order $O\left(g^{2}\right)$ ], through Eq. (11) we obtain strong coupling corrections to the Carnot efficiency.

Limit of large S.-Let us briefly discuss the macroscopic limit, in which $S$ becomes large. The correcting terms to work and heat in Eqs. (4) and (12) can be bounded by the interaction strength as $\Delta F_{\min }^{(\mathrm{res}) /(\mathrm{irr})} \leq 2\|V\|, T I\left(\omega^{(N)} ; S: B\right) \leq$ $2\|V\|$, where $\|V\|$ is the operator norm of $V$. The first bound is derived in Sec. II D of Ref. [46], whereas the second one follows from Ref. [65]. For many-body systems with local interactions, $\|V\|$ scales as the boundary between $S$ and $B$, while $W$ and $Q$ scale as the volume of $S$. Thus, the above corrections become negligible in the limit of large systems. In other words, macroscopic phenomenological thermodynamics is insensitive to the strength of the underlying interactions, making these effects only relevant for small systems.

Power.-Although nonzero interactions between $S$ and $B$ tend to increase dissipation, they can enhance power by decreasing the time scale of thermalization $\tau$. We now derive an upper bound for such a power enhancement in a Carnot-like engine. In order to do so, we need some considerations on how $\tau$ is related to $g$. A dimensional analysis argument suggests that $\tau \propto g^{-1}$. In fact, in Sec. VI A of the Supplemental Material [46] we show that $\tau \geq \delta Q / g r$, with $\delta Q$ being the energy change of $B$ during the equilibration and $r:=\left\|\left[H_{B}, V\right]\right\|$ the maximum rate in which $S$ and $B$ can exchange energy. From these considerations we obtain (see Sec. VIB of the Supplemental Material [46])

$$
P:=\frac{W}{\Delta t} \leq \frac{g r_{c} \eta(g)}{1-\eta+r_{c} / r_{h}}<g r_{h} \eta(g),
$$

where $W$ is the work produced in a cycle, $\Delta t$ the cycle time, $\eta$ the efficiency of the machine, $r_{c / h}:=\left\|\left[H_{B_{c / h}}, V_{c / h}\right]\right\|$, and
$V_{c / v}$ couples $S$ to the cold or hot bath. Using Eq. (14) we can expand Eq. (15) in $g$, yielding $P(g) \leq g r_{c} \eta^{\mathrm{C}} /$ $\left(1-\eta^{\mathrm{C}}+r_{c} / r_{h}\right)-O\left(g^{3}\right)$.

While the bound (15) is valid for arbitrary systems, it is expected to be very crude in general, as it depends on $\|V\|$. Further progress relies on a better characterization of the time scales of equilibration of generic systems, a notoriously hard and diverse problem [1]. Relations between power and efficiency of Carnot engines have also been obtained in Refs. [66-68], yielding complementary results.

Thermodynamic protocols within the CL model.-We now apply our findings in the paradigmatic quantum Brownian motion, captured in the Ullersma or CaldeiraLeggett (CL) model [69,70]. In this model, $H=H_{S}+g V+H_{B}+H_{L}$, where $S$ is a harmonic oscillator, $H_{S}=\left(m \omega^{2} x^{2}+p^{2} / m\right) / 2, \quad B$ a bosonic bath, $H_{B}=\sum_{k}\left(m_{k} \omega_{k}^{2} x_{k}^{2}+p_{k}^{2} / m_{k}\right) / 2$, coupled to $S$ through $g V$ with $V=x \sum_{k} g_{k} x_{k}$ and where $g$ quantifies the strength of the interaction and, for Ohmic spectral densities, the deviations from Markovian dynamics [71], and, finally, $H_{L}=x^{2} g^{2} \sum_{k} g_{k}^{2} /\left(m_{k} \omega_{k}^{2}\right)$ is a renormalization term. This model plays a crucial role in open quantum systems [61] and finds numerous applications in thermodynamics $[12,13,15,18,20,29,72-75]$.

The equilibrium state of $S$ in the CL-model is given by $\operatorname{Tr}_{B}\left[\omega_{\beta}(H)\right]$, thus satisfying Eq. (3) $[4,76,77]$. Furthermore, the total Hamiltonian is quadratic, and, hence, can be solved exactly with matrices of order $O\left(n^{2}\right)$, where $n$ is the number of oscillators in $B$ (see, e.g., Refs. [57,58]). This allows us to numerically simulate thermodynamic protocols exactly for arbitrary strong coupling and large (but finite) baths. Details on the discretization of the CL model and its simulation are provided in Sec. VII of the Supplemental Material [46]. There, the equilibration time is also discussed, finding $\tau \propto 1 / g^{2}$, for $g \leq 1$, using techniques from Refs. [50,51], which agrees with standard perturbative approaches [61].

Moving to work-extraction protocols, we stress that the first order corrections in Eqs. (9) and (10) vanish, as the thermal state of $H_{B}$ is symmetric under $x_{k} \leftrightarrow-x_{k}$. This implies that the optimal protocol in the weak coupling regime is in fact also optimal for small but nonzero $g$. This is perfectly illustrated in Fig. 1, where we plot the work extracted using the weak coupling protocol and the optimal one, which is obtained by numerically minimizing $\Delta F^{(\text {res }) /(\text { irr })}$. Differences between the two start appearing only at higher orders than $O\left(g^{2}\right)$. Note also that Fig. 1 shows an excellent agreement between the exact unitary dynamics and our framework, in which Eq. (3) is assumed, even when many quenches are performed. For studying power, we keep the number of quenches $N$ fixed and vary the coupling strength $g$. As a result of the equilibration time $\tau \propto 1 / g^{2}$ for $g \leq 1$, the power $P(g)=W(g) / \tau(g)$, scales as $P(g) \propto g^{2} W^{\text {(weak) }}-O\left(g^{3}\right)$, as shown in the inset of Fig. 1. 


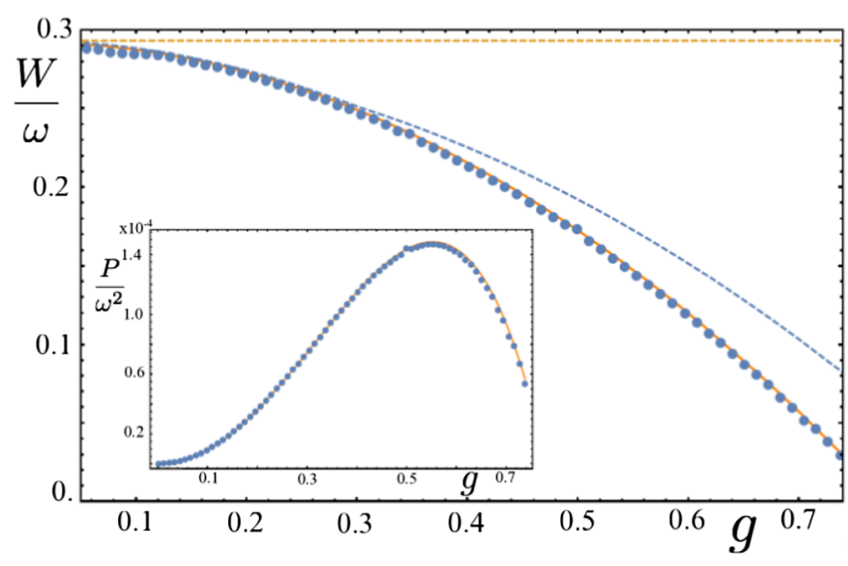

FIG. 1. We take $\rho^{(0)}=\omega_{\beta_{S}}\left(H_{S}\right) \otimes \omega_{\beta}\left(H_{B}\right)$, with $\beta_{S} \neq \beta$, and consider the protocol for maximal work extraction in the weak coupling regime: A quench $\omega \mapsto \omega \beta / \beta_{S}, m \mapsto \beta_{S} / \beta$, followed by an isothermal process back to $H_{S}$. We model the isothermal process by $N=200$ quenches, with a waiting time $10 / \mathrm{g}^{2}$ when computing the unitary dynamics. Outer: Work vs strength of interaction. (i) Blue dots: extracted work calculated through the unitary evolution of $S B$, (ii) orange line: same computation but assuming (3), (iii) dashed blue: optimal protocol by numerically optimising $W$, (iv) dashed orange: $W^{(\text {weak })}$ (i.e., $g=0$ ). Inner: Power vs interaction. Blue dots: exact unitary evolution. Orange line: effective description using our framework. Parameters for both figures: Setting $\omega=1, B$ consists of $n=165$ oscillators with $m_{k}=1$ and the $\omega_{k}$ 's uniformly distributed up to $\Omega=1.2$, and $\beta=3.5$. For $S, \beta_{S}=1$, and $m=1$.

Conclusion.-By bringing insights from the theory of equilibration in closed many-body systems to the realm of quantum thermodynamics, we have derived general strong coupling corrections to the second law of thermodynamics. These corrections are applicable to any model of interest, and have been obtained by designing optimal thermodynamic protocols in the strong coupling regime. They become relevant if the working body is a small system, and vanish in first order with the interaction strength. An upper bound on the power enhancement due to the interaction strength has also been derived. A particularly relevant open problem is to extend these considerations to scenarios where $S$ is simultaneously strongly coupled to more than one thermal bath. Then reaction coordinate mappings $[12,13]$ appear as a promising technique to extend results in the weak coupling [78]. It is the hope that this work further stimulates the emerging field of strong-coupling quantum thermodynamics, aiming at identifying the potential and burden coming along with such interactions.

We thank I. Cirac, A. Molnar, and T. Shi for insightful discussions. We also thank P. Strasberg, G. Schaller, and D. Gelbwaser-Klimovsky for useful comments on the manuscript. H. W. acknowledges funding from the ERC (TAQ) and the Studienstiftung des Deutschen Volkes, M. P. L. from the Alexander von Humboldt foundation, R. G. from the DFG (GA 2184/2-1). J.E. thanks for support by the
DFG (CRC 183, EI 519/7-1, EI 519/14-1), the ERC (TAQ), the EU (AQuS), and the Templeton Foundation. A. R. acknowledges the Spanish Ministry MINECO (National Plan 15 Grant: FISICATEAMO No. FIS2016-79508-P, SEVERO OCHOA No. SEV-2015-0522), Fundació Cellex, Generalitat de Catalunya (AGAUR Grant No. 2017 SGR 1341, SGR 875 and CERCA/Program), ERC AdG OSYRIS, EU FETPRO QUIC, and the CELLEX-ICFO-MPQ fellowship.

[1] C. Gogolin and J. Eisert, Rep. Prog. Phys. 79, 056001 (2016).

[2] M. Müller, E. Adlam, L. Masanes, and N. Wiebe, Commun. Math. Phys. 340, 499 (2015).

[3] F. G. S. L. Brandao and M. Cramer, arXiv:1502.03263.

[4] Y. Subasi, C. H. Fleming, J. M. Taylor, and B. L. Hu, Phys. Rev. E 86, 061132 (2012).

[5] T. Farrelly, F. G. S. L. Brandao, and M. Cramer, Phys. Rev. Lett. 118, 140601 (2017).

[6] S. Vinjanampathy and J. Anders, Contemp. Phys. 57, 545 (2016).

[7] J. Goold, M. Huber, A. Riera, L. d. Rio, and P. Skrzypczyk, J. Phys. A 49, 143001 (2016).

[8] R. Kosloff, Entropy 15, 2100 (2013).

[9] D. Gelbwaser-Klimovsky and A. Aspuru-Guzik, J. Phys. Chem. Lett. 6, 3477 (2015).

[10] R. Uzdin, A. Levy, and R. Kosloff, Entropy 18, 124 (2016).

[11] G. Katz and R. Kosloff, Entropy 18, 186 (2016).

[12] P. Strasberg, G. Schaller, N. Lambert, and T. Brandes, New J. Phys. 18, 073007 (2016).

[13] D. Newman, F. Mintert, and A. Nazir, Phys. Rev. E 95, 032139 (2017).

[14] D. Xu, C. Wang, Y. Zhao, and J. Cao, New J. Phys. 18, 023003 (2016).

[15] P. Hänggi, G.-L. Ingold, and P. Talkner, New J. Phys. 10, 115008 (2008).

[16] M. F. Gelin and M. Thoss, Phys. Rev. E 79, 051121 (2009).

[17] M. Campisi, P. Talkner, and P. Hänggi, Phys. Rev. Lett. 102, 210401 (2009).

[18] I. Kim and G. Mahler, Phys. Rev. E 81, 011101 (2010).

[19] M. Campisi, D. Zueco, and P. Talkner, Chem. Phys. 375, 187 (2010).

[20] S. Hilt, B. Thomas, and E. Lutz, Phys. Rev. E 84, 031110 (2011).

[21] C. K. Lee, J. Cao, and J. Gong, Phys. Rev. E 86, 021109 (2012).

[22] R. Gallego, A. Riera, and J. Eisert, New J. Phys. 16, 125009 (2014).

[23] C. K. Lee, J. Cao, and J. Gong, Sci. Rep. 5, 11787 (2015).

[24] M. Esposito, M. A. Ochoa, and M. Galperin, Phys. Rev. Lett. 114, 080602 (2015).

[25] M. Esposito, M. A. Ochoa, and M. Galperin, Phys. Rev. B 92, 235440 (2015).

[26] A. Bruch, C. Lewenkopf, and F. von Oppen, Phys. Rev. Lett. 120, 107701 (2018).

[27] M. Perarnau-Llobet, A. Riera, R. Gallego, H. Wilming, and J. Eisert, New J. Phys. 18, 123035 (2016).

[28] A. Kato and Y. Tanimura, J. Chem. Phys. 145, 224105 (2016). 
[29] T. G. Philbin and J. Anders, J. Phys. A 49, 215303 (2016).

[30] M. Carrega, P. Solinas, M. Sassetti, and U. Weiss, Phys. Rev. Lett. 116, 240403 (2016).

[31] U. Seifert, Phys. Rev. Lett. 116, 020601 (2016).

[32] C. Jarzynski, Phys. Rev. X 7, 011008 (2017).

[33] F. G. S. L. Brandao, M. Horodecki, J. Oppenheim, J. M. Renes, and R. W. Spekkens, Phys. Rev. Lett. 111, 250404 (2013).

[34] F. G. S. L. Brandao, M. Horodecki, N. H. Y. Ng, J. Oppenheim, and S. Wehner, Proc. Natl. Acad. Sci. U.S.A. 112, 3275 (2015).

[35] P. Cwiklinski, M. Studzinski, M. Horodecki, and J. Oppenheim, Phys. Rev. Lett. 115, 210403 (2015).

[36] M. Lostaglio, K. Korzekwa, D. Jennings, and T. Rudolph, Phys. Rev. X 5, 021001 (2015).

[37] H. Wilming, R. Gallego, and J. Eisert, Phys. Rev. E 93, 042126 (2016).

[38] Note that the interaction energy and the bath energy remain constant. Furthermore, quenches on $S$ can be similarly applied when the interaction $V$ is turned off, and in this case Eq. (2) is also valid.

[39] Indeed, in the specific situation of the system $S$ and its bath $B$ being translationally invariant, Ref. [5] shows that the reduced state of the infinite time average is close in 1-norm to $\rho_{S}=\operatorname{tr}_{B}\left[e^{-\beta H} / \operatorname{tr}\left(e^{-\beta H}\right)\right]$, once one perturbs a Gibbs state by acting with a completely positive map on it supported on a constant number of sites only. Here, in contrast, we consider Hamiltonian quenches in $S$. However, in the high temperature limit [40], the effect of such quenches can be approximated by completely positive maps acting on $S$ and a constant number of degrees of freedom of $B$. The closeness to the infinite time average is guaranteed for the overwhelming majority of times by invoking the equilibration results of Ref. [41] in conjunction to the bounds on the effective dimension from Ref. [5].

[40] M. Kliesch, C. Gogolin, M. J. Kastoryano, A. Riera, and J. Eisert, Phys. Rev. X 4, 031019 (2014).

[41] N. Linden, S. Popescu, A. J. Short, and A. Winter, Phys. Rev. E 79, 061103 (2009).

[42] Hamiltonian cyclicity means that in a protocol of $N+1$ steps, we have $H^{(N+1)}=H^{(0)}$, where $N$ can be arbitrarily large.

[43] R. Alicki, M. Horodecki, P. Horodecki, and R. Horodecki, Open Syst. Inf. Dyn. 11, 205 (2004).

[44] P. Strasberg and M. Esposito, Phys. Rev. E 95, 062101 (2017).

[45] J. Anders and V. Giovannetti, New J. Phys. 15, 033022 (2013).

[46] See Supplemental Material at http://link.aps.org/ supplemental/10.1103/PhysRevLett.120.120602, which includes Refs. [22,40,47-58] for detailed derivations and extended discussions of our results.

[47] H. Mori, J. Phys. Soc. Jpn. 11, 1029 (1956).

[48] R. Kubo, J. Phys. Soc. Jpn. 12, 570 (1957).

[49] H. Mori, Prog. Theor. Phys. 33, 423 (1965).

[50] T. R. Oliveira, D. Jonathan, C. Charalambous, M. Lewenstein, and A. Riera, New J. Phys., DOI:10.1088/1367-2630/ aab03b (2018).
[51] H. Wilming, M. Goihl, C. Krumnow, and J. Eisert, arXiv:1704.06291.

[52] D. Petz and G. Toth, Lett. Math. Phys. 27, 205 (1993).

[53] S. Amari and H. Nagaoka, Methods of Information Geometry, Translations of Mathematical Monographs (American Mathematical Society, Providence, 2007).

[54] J. Aberg, Nat. Commun. 4, 1925 (2013).

[55] W. Pusz and S. Woronowicz, Commun. Math. Phys. 58, 273 (1978).

[56] A. Lenard, J. Stat. Phys. 19, 575 (1978).

[57] A. Rivas, A. D. K. Plato, S. F. Huelga, and M. B. Plenio, New J. Phys. 12, 113032 (2010).

[58] J. Eisert, M. Cramer, and M. B. Plenio, Rev. Mod. Phys. 82, 277 (2010).

[59] A. Ferraro, A. García-Saez, and A. Acín, Europhys. Lett. 98, 10009 (2012).

[60] S. Hernández-Santana, A. Riera, K. V. Hovhannisyan, M. Perarnau-Llobet, L. Tagliacozzo, and A. Acín, New J. Phys. 17, 085007 (2015).

[61] U. Weiss, Quantum Dissipative Systems (World Scientific, Singapore, 1993).

[62] H. P. Breuer and F. Petruccione, The Theory of Open Quantum Systems (Oxford University Press, New York, 2002).

[63] More specifically, $H^{(N)}=H_{S}^{(N)}+V+H_{B}$ and $H^{(N+1)}=$ $H_{S}^{(N)}+H_{B}$.

[64] If one wishes to expand $Q(g)$ with respect to $Q^{\text {(weak) }}$, then the first order corrections do not vanish. This is discussed in more detail in the Supplemental Material [46].

[65] M. M. Wolf, F. Verstraete, M. B. Hastings, and J. I. Cirac, Phys. Rev. Lett. 100, 070502 (2008).

[66] A. E. Allahverdyan, K. V. Hovhannisyan, A. V. Melkikh, and S. G. Gevorkian, Phys. Rev. Lett. 111, 050601 (2013).

[67] N. Shiraishi, K. Saito, and H. Tasaki, Phys. Rev. Lett. 117, 190601 (2016).

[68] N. Shiraishi and H. Tajima, Phys. Rev. E 96, 022138 (2017).

[69] A. O. Caldeira and A. J. Leggett, Physica (Amsterdam) 121A, 587 (1983).

[70] P. Ullersma, Physica (Amsterdam) 32, 56 (1966).

[71] S. Groeblacher, A. Trubarov, N. Prigge, G. D. Cole, M. Aspelmeyer, and J. Eisert, Nat. Commun. 6, 7606 (2015).

[72] J. Eisert and M. B. Plenio, Phys. Rev. Lett. 89, 137902 (2002).

[73] A. E. Allahverdyan and T. M. Nieuwenhuizen, Phys. Rev. Lett. 85, 1799 (2000).

[74] T. M. Nieuwenhuizen and A. E. Allahverdyan, Phys. Rev. E 66, 036102 (2002).

[75] M. Carrega, P. Solinas, A. Braggio, M. Sassetti, and U. Weiss, New J. Phys. 17, 045030 (2015).

[76] V. Ambegaokar, Ann. Phys. (Amsterdam) 16, 319 (2007).

[77] Numerical evidence also shows that the energy contained in the interaction $V$ also equilibrates to the reduced of a global thermal state.

[78] N. Brunner, N. Linden, S. Popescu, and P. Skrzypczyk, Phys. Rev. E 85, 051117 (2012). 